\title{
Stability and Practically Stability of Impulsive Integro-Differential Systems by Cone-Valued Lyapunov Functions
}

\author{
Zhuoying Lv*, Guocheng Gao and Lailiang Zhang
}

Public Course Department, Shandong University of Science and Technology, Jinan, Shandong, 250031, P.R. China

\begin{abstract}
Stability and practically stability comparison criteria of impulsive integro-differential systems with fixed moments of impulse effects are established by cone-Lyapunov functions through comparing with impulsive ordinary differential equations.
\end{abstract}

Keywords: Impulsive Integro-Differential System; Cone-Lyapunov Function; Stability

\section{Introduction}

Impulsive integro-differential systems which are an important embranchment of nonlinear impulsive differential systems [1], arise from extensive applications in nature-science such as mathematic models of circuit simulation in physics and neuronal networks in biology. Consequently there are some results about stability of such systems by vector Lyapunov functions coupled with Razumikhin techniques $[2,3]$. However, it's difficult to choose a right vector Lyapunov function because of the restrict conditions. At the same time, the method of cone-valued Lyapunov functions is well known to be advantageous in applications [4]. Hence, the stability results for impulsive integro-differential systems could be improved via the method of cone-valued Lyapunov functions.

Adeyeye [5] considered the comparison principle by cone-valued Lyapunov functions for a class of integro-differential system without impulses. But it was not proved and also cannot be applied to impulsive integrodifferential systems. In this paper we shall firstly prove the comparison principle. Then by employing cone-valued Lyapunov functions a new comparison principle for impulsive integro-differential systems with fixed moments of impulse effects is established, which is compared with impulsive differential systems whose stability is relatively easy to solve. Finally the relevant new comparison criteria of stability and practically stability [6] of impulsive integro-differential systems are obtained too.

The remainder of this paper is organized as follows. In section 2 , we describe impulsive integro-differential systems and introduce some notions and concepts. In section 3, we get some comparison results of stability and practically stability of the impulsive integro-differential systems with fixed moments of impulse effects by using the method of cone-Lyapunov functions.

\section{Preliminaries} form

Consider the following impulsive integro-differential systems of the

$$
\left\{\begin{array}{c}
x^{\prime}=f(t, x, T x), \\
x\left(t_{k}\right)=J_{k}\left(x\left(t_{k}^{-}\right)\right), \\
x\left(t_{0}^{+}\right)=x_{0},
\end{array}\right.
$$

where $\mathrm{N}$ is the set of all positive integers, $0<\mathrm{t}_{1}<\mathrm{t}_{2}<\ldots<\mathrm{t}_{\mathrm{k}}<\ldots$ and $t_{k} \rightarrow \infty(k \rightarrow \infty) . \quad f \in C\left(\left[t_{k}, t_{k}+1\right) \times S(\rho) \times R^{n}, R^{n}\right)(k \in N), f(t, 0,0) \equiv 0, \quad$ where $S(\rho)=\left\{x:|x|<\rho, x \in R^{n}\right\}$.

$T x=\int_{t_{0}}^{t} K(t, s, x(s)) d s$, where $K \in C\left(\left[t_{k}, t_{k+1}\right) \times\left[t_{k}, t_{k+1}\right) \times S(\rho), R^{n}\right), K(t, t, 0) \equiv 0$.

$J_{k}(x): S(\rho) \rightarrow R^{n}, J_{k}(0) \equiv 0(\forall k \in N)$ and there exists $\rho_{1}: 0<\rho_{1} \leq \rho$ such that $x \in S\left(\rho_{1}\right)$ implies that $J_{k}(x) \in S(\rho)$ for all $k \in N$.

In addition we always assume that $\mathrm{f}_{\mathrm{k}} \mathrm{J}_{\mathrm{k}}$ satisfy certain conditions such that the solution of system (1)exists on $\left[t_{0},+\infty\right]$ and is unique. We denote by $x(t)=x\left(t, t_{0}, x_{0}\right)$ the solution of system (1) with initial value $\left(t_{0}, x_{0}\right)$. Since $f(t, 0,0)=0, J_{k}(0)=0, k \in N$, then $\mathrm{x}(\mathrm{t})=0$ is a solution of $(1)$, which is called the trivial solution. Note that the solutions $\mathrm{x}(\mathrm{t})$ of $(1)$ are right continuous, satisfying $x\left(t_{k}^{+}\right)=x\left(t_{k}\right)=J_{k}\left(x\left(t_{k}^{-}\right)\right)$.

Let $\mathrm{t}_{0}=0$; the following sets are introduced:

$$
G_{k}=\left\{(t, x) \in R_{+} \times S(\rho): t_{k-1}<t<t_{k}\right\}, G=\bigcup_{k=1}^{\infty} G_{k}
$$

For convenience, we define the following classes of functions:

$K=\left\{a \in C\left[R_{+}, R_{+}\right]:\right.$strictlyincrea $\sin g$ and $\left.a(0)=0\right\}$ strictly increasing and $\mathrm{a}(0)=0 \mathrm{~g}$;

$$
\begin{aligned}
& C K=\left\{a \in C\left[R_{+}^{2}, R_{+}\right]: \text {for every } t \in R_{+}, a(t, s) \in K\right\} \\
& S(h, \rho)=\left\{(t, x) \in R_{+} \times R^{n}: h(t, x)<\rho\right\}
\end{aligned}
$$

In addition, we introduce some definitions as follows:

Definition 1: Let $Z \subseteq R^{n}$ be a cone, that is, $Z$ is closed, convex with $\lambda Z \subset Z, \lambda \geq 0$ and $Z \cap(-Z)=\{0\}$ with interior $Z^{0} \neq \phi$. For any $x, y \in R^{n}$, we let $x \leq y$ if $y-x \in Z$ and for any functions $u, v: R_{+} \rightarrow R^{n}, u \leq v$ if $u(t) \leq v(t)$ on $R_{+}$. Also let $Z^{*}=\left\{\varphi \in R^{n}, \varphi(x) \geq 0\right.$, for all $\left.x \in Z\right\}$ and $Z_{0}^{*}=Z^{*}-\{0\}$,

*Corresponding author: Zhuoying Lv, Public Course Department, Shandong University of Science and Technology, Jinan, Shandong, 250031, P.R. China E-mail: fancygreen@126.com

Received July 17, 2013; Accepted September 16, 2013; Published October 10 2013

Citation: Lv Z, Gao G, Zhang L (2013) Stability and Practically Stability of Impulsive Integro-Differential Systems by Cone-Valued Lyapunov Functions. J Appl Computat Math 2: 141. doi:10.4172/2168-9679.1000141

Copyright: @ 2013 Lv Z, et al. This is an open-access article distributed under the terms of the Creative Commons Attribution License, which permits unrestricted use, distribution, and reproduction in any medium, provided the original author and source are credited. 
where $\varphi(x)=\sum_{i=1}^{n} \varphi_{i} x_{i}$

Definition 2: A function $F: R^{n} \rightarrow R^{n}$ is said to be quasi-monotone nondecreasing relative to the cone $Z \subseteq R^{n}$ if $x \leq y$ and $\varphi(y-x)=0$ for all $\varphi \in Z_{0}^{*}$ implies $\varphi(F(y)-F(x)) \geq 0$.

Definition 3: We shall say that the function $V_{0}: R_{+} \times R^{n} \rightarrow Z$ belongs to the class of cone-Lyapunov if $\backslash$

i. $V \in V_{0}$ is continuous on $G_{k} \times R^{n}$ and the following limits exist $\lim _{(t, y) \rightarrow\left(t_{k}^{,}, x\right)} V(t, y), k \in N$

ii. $V(t, x) \in V_{0}$ is locally Lipschitz in $\mathrm{x}$ relative to $\mathrm{Z}$.

Derivative of $V(t, x) \in V_{0}$ along system(1) is defined:

$$
D^{-} V(t, x(t))=\underset{h \rightarrow 0^{-}}{\limsup } \frac{1}{h}[V(t+h, x(t)+h f(t, x(t)))-V(t, x(t))] .
$$

Definition 4: The trivial solution of (1) is said to be

i. stable, if for any $\varepsilon>0$, every $t_{0} \in R_{+}$, there exists a $\delta=\delta\left(t_{0}, \varepsilon\right)>0$ such that $\left|x_{0}\right|<\delta$ implies $\left|x\left(t, t_{0}, x_{0}\right)\right| \leq \varepsilon$ for all $t \geq t_{0}$;

ii. uniformly stable, if $\delta$ in (i) is independent on $t_{0}$;

iii. uniformly asymptotically stable, if it is uniformly stable, and there exists a such that for any and every $t_{0} \in R_{+}$there is a $T=T(\varepsilon)>0$ such that $\left|x_{0}\right|<\delta$ implies $\left|x\left(t, t_{0}, x_{0}\right)\right| \leq \varepsilon$ for all $t \geq t_{0}+T$

Definition 5: The trivial solution of (1) is said to be

i. practically stable, if for given number pair $(\lambda, A)$ with $0<\lambda<A$, we have $\left|x_{0}\right|<\lambda$ implies $|x(t)|<A, t \geq t_{0}$ for some $t_{0} \in R_{+}$;

ii. uniformly practically stable, if(i)holds for every $t_{0} \in R_{+}$;

iii. practically quasi-stable, if for given number $(\lambda, B, T)>0$ and some $t_{0} \in R_{+}$, we have $\left|x_{0}\right|<\lambda$ implies $|x(t)|<B$ for $t \geq t_{0}+T$;

iv. uniformly practically quasi-stable, if (iii) holds for every $t_{0} \in R_{+}$; v. strongly practically stable, if (i) and (iii) hold together;

vi. strongly uniformly practically stable, if (ii) and (iv) hold together. We also consider the comparison differential system:

$$
\left\{\begin{array}{c}
u^{\prime}=g(t, u), \quad t \neq t_{k} \\
u\left(t_{k}\right)=\Psi_{k}\left(u\left(t_{k}^{-}\right)\right) \quad k \in N \\
u\left(t_{0}^{+}\right)=u_{0}
\end{array}\right.
$$

where $g \in C\left(\left[t_{k}, t_{k}+1\right) \times Z, R^{n}\right), k \in N$.

In addition we always assume that $g, \Psi_{k}$ satisfy certain conditions such that the solution of system(2) exists on $\left[t_{0}+\infty\right]$ and is unique. We denote by $u(t)=u\left(t, t_{0}, u_{0}\right)$ the solution of system(2) with initial value $\left(t_{0}, u_{0}\right)$. Note that the solutions $u(t)$ of (2) are right continuous, satisfying $u\left(t_{k}^{+}\right)=u\left(t_{k}\right)=\Psi_{k}\left(x\left(t_{k}^{-}\right)\right)$.

Definition 6: The trivial solution of (2) is said to be

i. $\phi_{0}$-stable, if for any $\varepsilon>0$, every $t_{0} \in R_{+}$, there exists a $\delta=\delta\left(t_{0}, \varepsilon\right)>0$ such that $\left(\phi_{0}, u_{0}\right)<\delta$ implies $\left(\phi_{0}, u(t)\right)<\varepsilon$, for all $t \geq t_{0}$; where $\phi_{0} \in Z_{0}^{*}$;

ii. $\phi_{0}$ - uniformly stable, if $\delta$ in (i) is independent on $t_{0}$;

iii. $\phi_{0}$-attractive, if for any $\varepsilon>0$, every $t_{0} \in R_{+}$, there exists a $\delta=\delta\left(t_{0}\right)>0, T=T\left(t_{0}, \varepsilon\right)>0$, such that $\left(\phi_{0}, u_{0}\right)<\delta$ implies for all $t \geq t_{0}+T$, where $\phi_{0} \in Z_{0}^{*}$;

iv. $\phi_{0}$ - uniformly attractive, if $\delta, T$ in(iii)is independent on $t_{0}$;

v. $\phi_{0}$-asymptotically stable, if (i)and(iii) hold together;

vi. $\phi_{0}$ - uniformly asymptotically stable, if (ii) and (iv) hold together.

Definition 7: The trivial solution of (2) is said to be

i. $\phi_{0}$ - practically stable, if for given number pair $(\lambda, A)$ with $0<\lambda<A$, we have $\left(\phi_{0}, u_{0}\right)<\lambda$ implies $\left(\phi_{0}, u(t)\right)<A, t \geq t_{0}$ for some $t_{0} \in R_{+}, \phi_{0} \in Z_{0}^{*}$, where $\phi_{0} \in Z_{0}^{*}$;

ii. $\phi_{0}$ - uniformly practically stable, if(i)holds for every $t_{0} \in R_{+}$;

iii. $\phi_{0}$-practically quasi-stable, if for given number $(\lambda, B, T)>0$ and some $t_{0} \in R_{+}, \phi_{0} \in Z_{0}^{*}$ we have $\left(\phi_{0}, u_{0}\right)<\lambda$ implies $\left(\phi_{0}, u(t)\right)<B$, for all $t \geq t_{0}+T$;

iv. $\phi_{0}$ - uniformly practically quasi-stable, if (iii) holds for every $t_{0} \in R_{+}$;

v. $\phi_{0}$-strongly practically stable, if (i) and (iii) hold together;

vi. $\phi_{0}$-strongly uniformly practically stable, if (ii) and (iv) hold together.

\section{Main Results}

Lemma 1: Assume that

i. $g \in C\left[R_{+} \times Z, R_{+}^{N}\right], g(t, u)$ is quai-monotone non decreasing in $u$ for each fixed $t$ on $Z$ and $r\left(t, t_{0}, u_{0}\right)$ is the maximal solution of the system $\left\{\begin{array}{c}u^{\prime}(t)=g(t, u), \\ u\left(t_{0}\right)=u_{0}\end{array}\right.$

ii. $V \in C\left[R_{+} \times R^{n}, Z\right], V(t, x)$ is locally Lipschitz in $\mathrm{x}$ relative to the cone $\mathrm{Z}$ and $D^{-} V(t, x(t)) \leq_{\mathrm{Z}}$

$g(t, V(t, x(t)))\left(t \geq t_{0}\right)$ for any solution $x(t)=x\left(t, t_{0}, x_{0}\right)$ of $\left\{\begin{array}{c}x^{\prime}=f(t, x, T x) \\ x\left(t_{0}\right)=x_{0} .\end{array}\right.$

Then $V(t 0, x 0) \leq_{\mathrm{Z}} u_{0}$ implies $V(t, x(t)) \leq_{\mathrm{Z}} r\left(t, t_{0}, u_{0}\right), t \geq t_{0}$.

Proof: Let $x(t)=x\left(t, t_{0}, x_{0}\right)$ is any solution of system in (ii), satisfying $V(t 0, x 0) \leq_{\mathrm{Z}} u_{0}$.

Set $m(t)=V(t, x(t))$, for small enough $h<0$; from (ii) $V(t, x)$ is locally Lipschitz in $\mathrm{x}$ relative to the cone $\mathrm{Z}$, therefore $m(t+h)-m(t) \leq_{\mathrm{Z}} L\|x(t+h)-x(t)-h f(t, x, T x)\|+V(t+h, x+h f(t, x, T x))-V(t, x(t))$, when $h \rightarrow 0$ we have $D^{-} m(t) \leq_{\mathrm{Z}} D^{-} V(t, x(t)) \leq_{\mathrm{Z}} g(t, m(t))$. For small enough $\forall \varepsilon>0$, consider system $\left\{\begin{array}{c}u^{\prime}(t)=g(t, u)+\varepsilon \eta, \\ u\left(t_{0}\right)=u_{0}, t_{0} \in R_{+},\end{array}\right.$
where $\eta \in Z$.

The solution of it is, then we have.

To prove the conclusion we only need to prove

$m(t) \leq_{\mathrm{Z}} u(t, \varepsilon), t \geq t_{0}$. 
If it is not true, there exists $t_{1}>t_{0}$, such that $u\left(t_{1}, \varepsilon\right)-m\left(t_{1}\right) \in \partial Z$, and $u(t, \varepsilon)-m(t) \in Z^{0}, t \in\left[t_{0}, t_{1}\right)$.

From (i) $g(t, u)$ is quai-monotone non decreasing in $\mathrm{u}$ for each fixed $t$ on $Z$, so there exists $\varphi \in Z_{0}^{*}$, such that

$$
\varphi\left(u\left(t_{1}, \varepsilon\right)-m\left(t_{1}\right)\right)=0 \text { and } \varphi\left(g\left(t_{1}, u\left(t_{1}, \varepsilon\right)\right)-g\left(t_{1}, m\left(t_{1}\right)\right)\right) \geq 0 .
$$

Set $\bar{u}(t)=\varphi(u(t, \varepsilon)-m(t)), t \in\left[t_{0}, t_{1}\right]$. Obviously $\bar{u}(t)>0, t \in\left[t_{0}, t_{1}\right)$ and $\bar{u}\left(t_{1}\right)=0$.

Therefore, $D^{-} \bar{u}\left(t_{1}\right)<0$

But

$$
\begin{aligned}
D^{-} \bar{u}\left(t_{1}\right) & =\varphi\left(D^{-} u\left(t_{1}, \varepsilon\right)-D^{-} m\left(t_{1}\right)\right) \\
& >\varphi\left(g\left(t_{1}, u\left(t_{1}, \varepsilon\right)\right)+\varepsilon \eta-g\left(t_{1}, m\left(t_{1}\right)\right)\right) \\
& >\varphi\left(g\left(t_{1}, u\left(t_{1}, \varepsilon\right)\right)-g\left(t_{1}, m\left(t_{1}\right)\right)\right) \\
& \geq 0,
\end{aligned}
$$

a contradiction So (3.1) holds, thus lemma 1 holds

\section{Lemma 2: Assume that}

i. $g \in C\left[\left[t_{k}, t_{k}+1\right) \times Z, R_{+}^{n}\right], k \in N, g(t, u) \quad$ is quai-monotone non decreasing in $\mathrm{u}$ for each fixed $\mathrm{t}$ on the cone $\mathrm{Z}$ and $r(t)=r\left(t, t_{0}, u_{0}\right)$ is the maximal solution of system(2) on Z;

ii. $\Psi \quad Z \rightarrow Z(k \in N)$ is strictly increasing on Z;

iii. for any solution $x(t)=x\left(t, t_{0}, x_{0}\right)$ of system(1) and $V \in V_{0}$,

$$
\begin{aligned}
& D^{-} V(t, x(t)) \leq_{\mathrm{Z}} g(t, V(t, x(t))), t \neq t_{k}, k \in N \\
& \text { iv. } V\left(t_{k}, J_{k}\left(x\left(t_{k}^{-}\right)\right)\right) \leq_{\mathrm{Z}} \Psi_{k}\left(V\left(t_{k}^{-}, x\right)\right) \\
& \text { Then implies }
\end{aligned}
$$

Proof: For any $t_{0} \in R_{+}$, and $t_{0} \in\left[t_{k}, t_{k}+1\right)$, for some $k \geq 1$, we designate $t_{i}=t_{k+i}, i=1,2, \ldots$, for convenience, then for $t \in\left[t_{0}, t_{1}\right)$ from lemma 1 we have $V(t, x(t)) \leq_{\mathrm{Z}} r_{1}\left(t, t_{0}, u_{0}\right)$ where $r_{1}\left(t, t_{0}, u_{0}\right)$ is the maximal solution of system $(2)$ on $\left[t_{0}, t_{1}\right)$ such that $r_{1}\left(t_{0}^{+}, t_{0}, u_{0}\right)=u_{0}$.

$$
\text { So } V\left(t_{1}^{-}, x\right) \leq_{\mathrm{Z}} r_{1}\left(t_{1}^{-}, t_{0}, u_{0}\right)
$$

Thus from (iii) we have

$$
\begin{aligned}
V\left(t_{1}, x\left(t_{1}, t_{0}, x_{0}\right)\right) & =V\left(t_{1}, J_{1}\left(x\left(t_{1}^{-}\right)\right)\right) \\
& \leq_{\mathrm{Z}} \Psi_{1}\left(V\left(t_{1}^{-}, x\right)\right) \\
& \leq_{\mathrm{Z}} \Psi_{1}\left(r_{1}\left(t_{1}^{-}, t_{0}, u_{0}\right)\right) \\
& =r_{1}\left(t_{1}^{-}, t_{0}, u_{0}\right) \doteq r_{1}
\end{aligned}
$$

Again from lemma 1, for $\mathrm{t} 2\left[\mathrm{t}_{1} ; \mathrm{t}_{2}\right), \mathrm{V}\left(\mathrm{t} ; \mathrm{x}\left(\mathrm{t} ; \mathrm{t}_{0} ; \mathrm{x}_{0}\right)\right) \leq \mathrm{zr} \mathrm{r}_{2}\left(\mathrm{t} ; \mathrm{t}_{1} ; \mathrm{r}_{1}\right)$; where $r_{2}\left(t ; t_{1} ; r_{1}\right)$ is the maximal solution of system (2) on $\left[t_{1} ; t_{2}\right)$ such that $r_{2}\left(t_{1}^{+}, t_{1}, r_{1}\right)=r_{1}$

Therefore we have

$$
V\left(t, x\left(t, t_{0}, x_{0}\right)\right) \leq z r_{k}\left(t, t_{k-1}, r_{k-1}\right),
$$

where $r_{k}\left(t, t_{k-1}, r_{k-1}\right)$ is the maximal solution of $\operatorname{system(2)}$ on $\left(t_{k-1}, t_{k}\right)$ such that $r_{k}\left(t_{k-1}^{+}, t_{k-1}, r_{k-1}\right)=r_{k-1}$
So if we define

$$
u^{*}(t)=\left\{\begin{array}{cl}
u_{0} & \multicolumn{1}{c}{t=t_{0},} \\
r_{1}\left(t, t_{0}, u_{0}\right), & t \in\left[t_{0} t_{1}\right), \\
\mid r_{2}\left(t, t_{1}, r_{1}\right), & t \in\left[t_{1}, t_{2}\right), \\
r_{k}\left(t, t_{k-1}, r_{k-1}\right), & \ldots \ldots . .
\end{array}\right.
$$

Then $u^{*}(t)$ is the solution of system (2), and $\mathrm{V}(\mathrm{t}, \mathrm{x}(\mathrm{t})) \leq \mathrm{Z} u^{*}(t)$; since $\mathrm{r}\left(\mathrm{t} ; \mathrm{t}_{0} ; \mathrm{u}_{0}\right)$ is the maximal solution of system(2) on $\mathrm{Z}$, we get immediately

$$
V(t, x(t)) \leq z\left(t, t_{0}, u_{0}\right), t \geq t_{0} .
$$

Theorem 1: Assume that $\exists a, b \in K$ such that:

i) $b(|x|) \leq\left(\varnothing_{0}, V(t, x)\right) \leq a(|x|), x \in S(\rho)$;

ii) $g \in C\left[\left[t_{k}, t_{k+1}\right) \times Z, R_{+}^{n}\right], k \in N, g(t, u) \quad$ is quai-monotone nondecreasing in $\mathrm{u}$ for each fixed $\mathrm{t}$ on the cone $\mathrm{Z}$ and $g(t, 0) \equiv 0, r(t)=r\left(t, t_{0}, u_{0}\right)$ is the maximal solution of system $(2)$ on Z;

iii) $\psi_{k}: Z \rightarrow Z(k \in N)$ is strictly increasing on $Z \psi_{k}(0) \equiv 0$;

(iv) for any solution $x(t)=x\left(t, t_{0}, x_{0}\right) \in S(\rho)$ of system (1) and $V \in V_{0}$ we have $D^{-} V(t, x(t)) \leq z g(t, V(t, x(t))), t \neq t_{k}, k \in N$;

v) $V\left(t_{k}, J_{k}\left(x\left(t_{k}^{-}\right)\right)\right) \leq z \psi_{k}\left(V\left(t_{k}^{-}, x\right)\right), x \in S\left(\rho_{1}\right)$.

Then the $\varnothing$ stability properties of the trivial solution of system (2) imply the corresponding stability properties of the trivial solution of system (1).

Proof: For any $\epsilon: 0<\epsilon<p_{1}$ every set $V(t)=V(t, x(t))$.

Let the trivial solution of system (2) is $\varnothing$-stable then for $b(\epsilon)>0$ every $t_{0} \in R_{+}$, there

Exists $\delta_{1=} \delta_{1}\left(t_{0}, \in\right) \quad$ such that $0 \leq\left(\varnothing_{0}, u_{0}\right)<\delta_{1} \quad$ implies $\left(\varnothing_{0}, u\left(t, t_{0}, u_{0}\right)\right)<b(\in), t \geq t_{0}$.

Set $\delta_{2}=\delta_{2}(\epsilon)$ satisfying $a\left(\delta_{2}\right)<\delta_{1}$.

We define $\delta=\min \left\{\delta_{1}, \delta_{2}\right\}$.

Next, we claim that $\left|x_{0}\right|<\delta$ implies

$|x(t)|<\in, t \geq t_{0}$.

If it is not true, there exists a solution $\mathrm{x}(\mathrm{t})$ of system(1) such that $x\left(t_{0}, t_{0}, x_{0}\right)=0$ with $\left|x_{0}\right|<\delta$, then there exists $t^{*}>t_{0}$ such that $t_{k} \leq t^{*}<t_{k+1}(k \in N)$ satisfying $\left|x\left(t^{*}\right)\right| \geq \in$ and $|x(t)|<\in, t_{0} \leq t<t_{k}$.

From $\quad 0<\epsilon<\rho_{1} \quad$ we have $\left|x\left(t_{k}\right)\right|=\left|J_{k}\left(x\left(t_{k}^{-}\right)\right)\right|<\rho$, then there exists $\mid \bar{t} \in\left[t_{k}, t^{*}\right]$ satisfying $\in \leq|x(\bar{t})|<\rho$. From (iv) we have $D^{-} V(t) \leq z g(t, V(t)), t \in\left[t_{0}, t\right]$.

Set $u_{0}=V\left(t_{0}\right)$ thus from lemma 2 we get that $t \in\left[t_{0}, t\right]$ implies $V(t) \leq z r\left(t, t_{0}, u_{0}\right)$, where $r\left(t, t_{0}, u_{0}\right)$ is the maximal solution of system (2) such that $u\left(t_{0}, t_{0}, u_{0}\right)=u_{0}$.

And from (i) $\left(\varnothing_{0}, u_{0}\right)=\left(\varnothing_{0}, V\left(t_{0}\right)\right) \leq a\left(\left|x_{0}\right|\right)<a(\delta)<\delta_{1}$ so from the -stability of the

trivial solution of system(2) we have $\left(\varnothing_{0}, r,\left(t, t_{0}, u_{0}\right)\right)<b(\in), t \geq t_{0}$. Therefore $b(\in) \leq b(|x(\bar{t})|) \leq\left(\varnothing_{0}, V(\bar{t})\right)<\left(\varnothing_{0}, r\left(\bar{t}, t_{0}, u_{0}\right)\right)<b(\in)$, a contradiction Then $(3.2)$ 
holds, thus the trivial solution of system (1) is stable. If the trivial solution of system (2) is $\varnothing_{0}$-uniformly stable then it is clear that $\delta$ will be independent of $\mathrm{t}_{0}$ and thus we get the uniform stability of the trivial solution of system (1). Assume that the trivial solution of system (2) is $\varnothing_{0}$-symptotically stable, consequently we get that the trivial solution of system(1) is stable, then for $\in=\rho_{1}$, there exists $\delta_{0}^{*}=\delta\left(t_{0}, p_{1}\right)$, such that $\left|x_{0}\right|<\delta_{0}^{*}$ implies ||$x(t) \mid<\rho_{1,} t \geq t_{0}$ From (iv) we have $D^{-} V(t) \leq z g(t, V(t)), t \geq t_{0}$.

For any $\in: 0<\epsilon<\rho_{1}$ every $t_{0} \in R_{+}$, from the $\varnothing_{0}$-attractivity of the trivial solution

of system (2) we can get that for $b(\in)>0$, every $t_{0} \in R_{+}$, there exists $\delta_{10}=\delta_{10}\left(t_{0}\right)>0$ and $T=T\left(t_{0} \in\right)>0$, such that $0 \leq\left(\varnothing_{0}, u_{0}\right)<\delta_{10}$ implies $\left(\varnothing_{0}, u\left(t, t_{0}, u_{0}\right)\right)<b(\in), t \geq t_{0}+T$.

Set $\delta_{0}=\min \left\{\delta_{0}^{*}, a^{-1}\left(\delta_{10}\right)\right\}$.

For $\left|x_{0}\right|<\delta_{0}$, set $u_{0}=V\left(t_{0}\right)$ then from lemma 2 we have $\left.V(t) \leq z r\left(t, t_{0}, u_{0}\right)\right), t \geq t_{0}$

And from (i) $\left(\varnothing_{0}, u_{0}\right)=\left(\varnothing_{0}, V\left(t_{0}\right)\right) \leq a\left(\left|x_{0}\right|\right)<a\left(\delta_{0}\right)<\delta_{10}$ so from the $\varnothing_{0}$-attractivity

Of the trivial solution of system (2) we get that $\left.\varnothing_{0}, r\left(t, t_{0}, u_{0}\right)\right)<b(\in), t \geq t_{0}+T \quad$ therefore $b(|x(t)|) \leq\left(\varnothing_{0}, V(t)\right) \leq\left(\varnothing_{0}, r\left(t, t_{0}, u_{0}\right)\right)>b(\in), t \geq t_{0}+T, \quad$ thus $\| x(t) \mid<\epsilon, t \geq t_{0}+T$ so the trivial solution of system(1) is attractive.

Then the trivial solution of system (1) is asymptotically stable. If the trivial solution of system (2) is $\varnothing_{0}$-uniformly asymptotically stable, then it is clear that $\delta_{0} \mathrm{~T}$ will be independent of t0, and thus we get the uniform asymptotically stability of the trivial solution of system (1).

Theorem 2: Assume that theorem1 (i)-(v) hold and we have:

(vi) for $0<\lambda<A \leq \rho_{1}$ with $a(\lambda)<b(A)$.

Then the $\varnothing_{0}$-practical stability properties of the trivial solution of system (2) with respect to $(a(\lambda), b(A))$ imply the corresponding practical stability properties of the trivial solution of system (1) with respect to $(\lambda, A)$.

Proof: Set $V(t)=V(t, x(t))$, suppose that the trivial solution of system (2) is $\varnothing_{0}$ practically

Stable with respect to $a(\lambda), b(A))$ then exists $t_{0} \in R_{+}$, such that $0 \leq\left(\varnothing_{0}, u_{0}\right)<a(\lambda)$ implies $\left(\varnothing_{0}, u\left(t, t_{0}, u\right)\right)<b(A), t \geq t_{0}$.

For above $t_{0} \in R_{+}$next we will prove that $\left|x_{0}\right|<\lambda$ implies $|x(t)|<A, t \geq t_{0}$ If it is not true, there exists a solution $\mathrm{x}(\mathrm{t})$ of system (1) such that $x\left(t_{0}, t_{0}, x_{0}\right)=0$ with $\left|x_{0}\right|<\delta$, then there exists $t^{*}>t_{0}$ such that $t_{k}>t^{*}<t_{k+1}(k \in N)$ satisfying $\left|x\left(t^{*}\right)\right| \geq A$ and $|x(t)|<A t_{0} \leq t<t_{k} \mid$.

Since $0<A<\rho_{1}$ we have $\left|x\left(t_{k}\right)\right|=\left|J_{k}\left(x\left(t_{k}^{-}\right)\right)\right|<\rho$ then exists $\bar{t} \in\left[t_{k}, t^{*}\right]$ satisfying $A \leq|x(\bar{t})|<\rho$.

From (iv) we have $D^{-} V(t) \leq z g(t, V(t)), t \in\left[t_{0}, t\right]$.

Set $u_{0}=V\left(t_{0}\right)$ then from lemma 2 we get that $t \in\left[t_{0}, t\right]$ implies $V(t) \leq z r\left(t, t_{0}, u_{0}\right)$, where

$r\left(t, t_{0}, u_{0}\right)$ is the maximal solution of system(2) such that $u\left(t_{0}, t_{0}, u_{0}\right)=u_{0}$. And from $\left(\varnothing_{0}, u_{0}\right)=\left(\varnothing_{0}, V\left(t_{0}\right)\right) \leq a\left(\left|x_{0}\right|\right)<a(\lambda)$, so from the $\varnothing_{0}$-practical stability we have $\left(\varnothing_{0}, r\left(t_{0}, t_{0}, u_{0}\right)\right)<b(A), t \geq t_{0}$.
Then $b(A) \leq b(|x(\bar{t})|) \leq\left(\varnothing_{0}, V(\bar{t})\right)<\left(\varnothing_{0}, r\left(\bar{t}, t_{0}, u_{0}\right)\right)<b(A)$ a contradiction thus (3.3) holds, so the trivial solution of system(1) is practically stable. Suppose that the trivial solution of system (2) is $\varnothing_{0}$-uniformly practically stable with respect to $(a(\lambda), b(A))$ then it is clear that $\mathrm{t}_{0}$ will be independent of $\mathrm{t}_{0}$, and thus we get the trivial solution of system (1) is uniformly practically stable with respect to $(\lambda, A)$. Suppose that the trivial solution of system (2) is $\varnothing_{0}$-strongly practically stable with respect To $(a(\lambda), b(A), b(B), T)$, consequently we get that the trivial solution of system (1) is practically stable with respect to $(\lambda, A)$ then exists $t_{0} \in R_{+}$, such that $\left|x_{0}\right|<\lambda$ implies $|x(t)|<A \leq \rho, t \geq t_{0}$. From (iv) $D^{-} V(t) \leq z g(t, V(t)), t \geq t_{0}$. And since the trivial solution of system(2) is $\varnothing_{0}$-practically quasi-stable with respect to $(a(\lambda), b(B), T)$, we have that $0 \leq\left(\varnothing_{0}, u_{0}\right)<a(\lambda)$ implies $\left(\varnothing_{0}, u\left(t, t_{0}, u_{0}\right)\right)<b(B), t \geq t_{0}+T$.

For $\left|x_{0}\right|<\lambda$ set $u_{0}=V\left(t_{0}\right)$ then from lemma 2 we get $\left.V(t) \leq z r\left(t, t_{0}, u_{0}\right)\right), t \geq t_{0}$.

And from (i) $\quad\left(\varnothing_{0}, u_{0}\right)=\left(\varnothing_{0}, V\left(t_{0}\right)\right) \leq a\left(\left|x_{0}\right|\right)<a(\lambda), \quad$ so $\quad$ from the $\varnothing_{0}$ practically quasistability of the trivial solution of system (2) we have $\left(\varnothing_{0}, r\left(t, t_{0}, u_{0}\right)\right)<b(B), t \geq t_{0}+T$ then $b(|x(t)|) \leq\left(\varnothing_{0}, V(t)\right) \leq\left(\varnothing_{0}, r\left(t, t_{0}, u_{0}\right)\right)<b(B), t \geq t_{0}+T \quad$ thus $|x(t)|<(B), t \geq t_{0}+T$ so the trivial solution of system (1) is practically quasi-stable with respect to $(\lambda, B, T)$.

Therefore the trivial solution of system (1) is strongly practically stable with respect to $(\lambda, A, B, T)$.

If the trivial solution of system (2) is $\varnothing_{0}$-strongly uniformly practically stable with respect to $(\lambda, A, B, T)$ then it is clear that the above proof establish for every $\mathrm{t}_{0}$, therefore we get the trivial solution of system (1) is strongly uniformly practically stable with respect to $(\lambda, A, B, T)$

\section{References}

1. Xilin F, Baoqiang Y, Yansheng L (2005) Theory of Impulsive Differential Systems. Science Press, Beijing of China 6.

2. Xilin F, Xinzhi L (1999) Uniform boundedness and stability criteria in terms of two measures for impulsive integero differential equations. Appl Math Comp 102: $237-255$.

3. Lv Zhuo-ying, Zhang Lai-liang, Liu Xiao-yan (2013) New Razumikhin-type Theorem on Stability of Zero Solution for Impulsive Integro-Differential Systems, Mathematics in Practice and Theory 43: 220-225.

4. Sun XH (2004) Stability Theory for Functional Differential Systems with Impulses at Fixed Times. Shandong Normal University, Shandong of China.

5. Adeyeye JO (2001) Cone-valued Lyapunov functions and stability in two measures for integro-differential systems. Nonl Anal 47: 4835-4843.

6. Lakshmikantham V, Leela S and Martynyuk AA (1990) Practical Stability of Nonliear Systems. World Scientific, Singapore. 